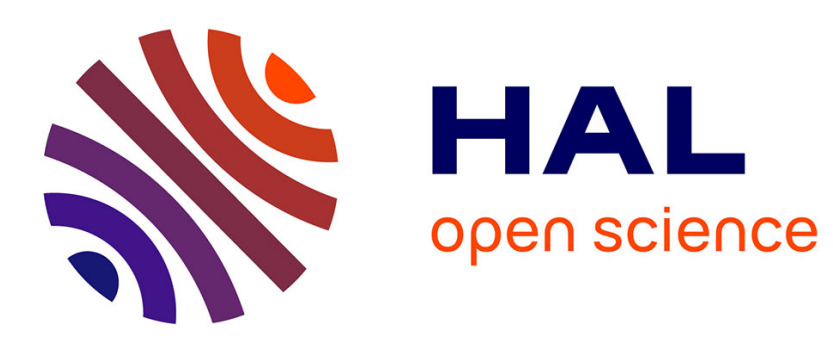

\title{
Entre coup de thâtre didactique et piège pédagogique: analyse d'une séance de préparation à l'épreuve écrite du baccalauréat
}

\author{
Christiane Descimon, Violaine Bigot
}

\section{To cite this version:}

Christiane Descimon, Violaine Bigot. Entre coup de thâtre didactique et piège pédagogique: analyse d'une séance de préparation à l'épreuve écrite du baccalauréat. Le Français Aujourd'hui, 2013, 181, pp.107-116. 10.3917/lfa.181.0107 . hal-01465762

\section{HAL Id: hal-01465762 https://hal.science/hal-01465762}

Submitted on 13 Feb 2017

HAL is a multi-disciplinary open access archive for the deposit and dissemination of scientific research documents, whether they are published or not. The documents may come from teaching and research institutions in France or abroad, or from public or private research centers.
L'archive ouverte pluridisciplinaire HAL, est destinée au dépôt et à la diffusion de documents scientifiques de niveau recherche, publiés ou non, émanant des établissements d'enseignement et de recherche français ou étrangers, des laboratoires publics ou privés. 


\section{ENTRE COUP DE THÉÂTRE DIDACTIQUE ET PIÈGE PÉDAGOGIQUE : ANALYSE D'UNE SÉANCE DE PRÉPARATION À L'ÉPREUVE ÉCRITE DU BACCALAURÉAT}

Christiane Descimon, Violaine Bigot

Armand Colin | «e français aujourd'hui »

2013/2 n $181 \mid$ pages 107 à 116

ISSN 0184-7732

ISBN 9782200928476

Article disponible en ligne à l'adresse :

http://www.cairn.info/revue-le-francais-aujourd-hui-2013-2-page-107.htm

\section{Pour citer cet article :}

Christiane Descimon, Violaine Bigot, " Entre coup de théâtre didactique et piège pédagogique : analyse d'une séance de préparation à l'épreuve écrite du baccalauréat », Le français aujourd'hui 2013/2 (n¹81), p. 107-116.

DOI 10.3917/lfa.181.0107

Distribution électronique Cairn.info pour Armand Colin.

(C) Armand Colin. Tous droits réservés pour tous pays.

La reproduction ou représentation de cet article, notamment par photocopie, n'est autorisée que dans les limites des conditions générales d'utilisation du site ou, le cas échéant, des conditions générales de la licence souscrite par votre établissement. Toute autre reproduction ou représentation, en tout ou partie, sous quelque forme et de quelque manière que ce soit, est interdite sauf accord préalable et écrit de l'éditeur, en dehors des cas prévus par la législation en vigueur en France. Il est précisé que son stockage dans une base de données est également interdit. 


\title{
ENTRE COUP DE THÉÂTRE DIDACTIQUE ET PIÈGE PÉDAGOGIQUE :
}

\section{ANALYSE D'UNE SÉANCE DE PRÉPARATION À L'ÉPREUVE ÉCRITE DU BACCALAURÉAT}

\author{
Christiane DESCIMON \\ \& \\ Violaine BIGOT \\ Université Sorbonne Nouvelle Paris 3 \\ Laboratoire DILTEC
}

Les enseignants conduisent leurs cours à partir de la planification qu'ils en ont faite mais aussi des "circonstances locales et singulières " (Fillietaz 2005) dans lesquelles se développe leur action. Les élèves jouent un rôle majeur dans ces "circonstances locales et singulières", et l'action qui se développe en classe appartient bien, malgré sa dimension unilatéralement planifiée, à la catégorie des « actions conjointes "(Vion 1992 : 98). Comment l'enseignant prend-il en compte cette dimension conjointe de l'action dans la planification qu'il peut en faire, comme dans le hic et nunc de l'échange?

Cette question sous-tend l'étude de ce cours de préparation à l'épreuve d'écriture d'invention, de l'épreuve anticipée de français (EAF) au baccalauréat. Les grandes étapes du déroulement de la séance seront rappelées avant que ne soit proposée une analyse focalisée sur la démarche de co-construction de savoirs que propose ce jeune enseignant, les difficultés qu'il rencontre ainsi que les solutions discursives inventées hic et nunc par les participants pour sortir d'un risque de conflit interactionnel.

\section{Présentation des données recueillies}

La séance analysée ci-après a été enregistrée dans une classe de seconde ${ }^{1}$. Au cours des deux séances précédentes, les élèves ont analysé une page d'une bande dessinée de Franquin visant à dénoncer l'absurdité de la peine de mort. Puis ils ont élaboré ensemble une «feuille de route » récapitulant les

\footnotetext{
1. C. Descimon a observé et vidéoscopé l'ensemble de la séquence. Elle a conduit et enregistré, une semaine après, un entretien d'autoconfrontation avec l'enseignant, puis, plus tard, une séance plus informelle de discussion avec les élèves. Elle s'est enfin chargée de l'ensemble des transcriptions. Les auteures de l'article sont très reconnaissantes à Jeremy d'avoir largement ouvert sa classe et pris en charge le montage des données vidéo. Elles remercient également les élèves de leur participation active.
} 
consignes et les contraintes d'écriture à respecter pour traiter le sujet suivant :

Rédigez du point de vue du bourreau l'histoire racontée par Franquin dans cette page de bande dessinée.

Pour la troisième séance, les élèves doivent rédiger chez eux un texte respectant les contraintes recensées collectivement : focalisation interne (le récit doit être à la première personne), cohérence narrative (c'est le bourreau qui raconte), présence de tous les éléments de la bande dessinée, humour noir de la dernière vignette (l'infinité des guillotines qui révèle que le bourreau va être exécuté à son tour).

\section{Présentation générale et chronologique de la séance}

\section{La planification d'un " coup de théâtre didactique "}

Quel est donc ce coup de théâtre qui a inspiré le titre de notre article?

Pendant cette séance, l'enseignant fait lire à chaque élève son texte qui est ensuite commenté par l'ensemble des camarades. Il découvre donc, en même temps que la classe, les productions des élèves, à deux exceptions près. Il explique en effet, lors de l'autoconfrontation, que « Al. et son copain V. [...] avaient donné leur feuille le mardi précédent [...] je me le gardais comme joker» (c.6) ${ }^{2}$.

Quel est ce joker que l'enseignant garde en réserve?

Pour raconter du point de vue du bourreau le contenu de la dernière vignette, l'élève $\mathrm{Al}$. a écrit: "Je me vis me faire trancher la tête, et j'entendis une voix qui disait... ". Phrase que l'enseignant considère comme inacceptable, puisqu' elle fait parler le bourreau après sa mort, et, de plus, au passé simple! Mais il n'en a rien dit à $\mathrm{Al}$. et espère que les autres élèves relèveront ce problème. Il a donc planifié cette séance autour de ce qu'il qualifie, pendant l'entretien de " coup de théâtre ». Pour résoudre ce problème, l'enseignant, à partir de la critique des textes rédigés par les élèves, veut faire formuler au groupe une contrainte qui n'était pas dans la feuille de route : le récit ne peut être qu'un récit en direct et doit donc être conduit au présent.

\section{La planification à l'épreuve de la gestion du cours}

Les textes d'élèves lus avant celui d'Al. pourraient permettre de traiter le " problème » énonciatif évoqué ci-dessus. Par exemple l'élève F. a écrit : "Aujourd'hui, je viens d'être nommé bourreau. C'est ma première exécution ». L'enseignant la félicite "Très bien, ça, très subtil... ", sans expliciter

2. (c.6) signifie que les propos de l'enseignant sont extraits du sixième commentaire effectué par l'enseignant lors de l'entretien d'autoconfrontation. 
ce qu'il trouve si «subtil ». Il ne met pas les élèves sur la voie, il veut attendre la lecture d'Al. pour les faire réfléchir à la question du régime temporel.

Lorsque Al. lit sa production, l'attention des autres élèves s'est un peu relâchée et le coup de théâtre se fait attendre : les élèves ne relèvent pas la phrase attendue par le professeur, mais pointent d'autres défauts.

Extrait $1^{3}$

107 Af : Il a dit « Désolé » fallait pas

108 Ens : Désolé pose un problème / pourquoi $\pi$ Qui dit ces paroles à la fin [...]

$112 \mathrm{Al}$ : Une voix.

113 Ens : Une voix + J'admire ta prudence [...]

116 Ens: Qui parle $\boldsymbol{\lambda}$ L'aumônier $\boldsymbol{\lambda}$ Le roi $\boldsymbol{\lambda}[\ldots]$ ça peut pas être quelqu'un d'extérieur à l'histoire c'est quelqu'un d'intérieur à l'histoire + Pourquoi ça peut pas être quelqu'un d'extérieur

L'enseignant, fidèle à sa logique de co-construction du savoir, prend en compte la proposition de l'élève et retarde la découverte de la phrase qui pose problème. Pour conduire l'attention des élèves vers cette phrase, il mène ensuite un questionnement serré :

\section{Extrait 2}

122 Ens : Quelque chose est gênant - dans ce qu'a fait Al. + veux-tu relire les dernières phrases s'il te plait

$123 \mathrm{Al}$ : (relecture) Je me vis me faire trancher la tête et j'entendis une voix qui disait $[\ldots]$

124 Ens : Bien + C'est quoi « je me vis trancher la tête et j'entendis une voix qui disait " + Quel est le problème

125 As : C'est bizarre

126 Ens : Si on se voit mourir on peut pas entendre sa voix

127 Af: On a un problème de cohérence $\pi$

128 Ens : On a +++ On a un problème de cohérence ++ Et là + un sujet comme celui-là + je vais vous donner maintenant un peu la clé de l'énigme [...] La difficulté majeure de ce sujet un peu vicieux autant vous le dire tout de suite, c'est qu'on vous demandait de faire parler quelqu'un qui raconte sa mort + Vous êtes d'accord $\boldsymbol{\lambda}$ Donc le texte doit s'arrêter nécessairement avec sa mort

L'enseignant parvient à faire dire aux élèves "C'est bizarre » et " on a un problème de cohérence ». Son silence, à la ligne 128 , fonctionne comme un potentiel marqueur de fin de tour parole. Mais les élèves ne poursuivent pas l'analyse et c'est lui qui, finalement, formule la conséquence en termes de contrainte d'écriture. L'exploitation pédagogique de la formulation de cette nouvelle contrainte se heurte à plusieurs formes de résistance.

Les élèves cherchent d'abord à l'esquiver :

3. Codes de transcription utilisés $\pi$ : intonation montante, + pause, +++ pause de plus de 5", MAJ : intonation forte. 


\section{Extrait 3}

135 Ens : Plus largement [...] comment ça se fait qu'on puisse lire le journal du bourreau qui va mourir $\pi$

$136 \mathrm{Am}$ : Il l'a écrit avant sa mort + Il savait qu'il allait se faire trancher la tête $[\ldots]$

137 Af : C'est pas lui qui l'a écrit c'est quelqu'un d'autre

138 Am : C'est le juge

139 Ens : C'est possible que ce soit pas lui $\pi$

140 Autre Af: C'est le bourreau d'après

141 Ens : Oui mais oublions ça $+\mathrm{Si}$ on vous dit de rédiger à la première personne il faut le faire +

Lorsque l'enseignant leur rappelle en ligne 141 une des contraintes recensées dans la feuille de route, les élèves ne contestent plus. Ils coopèrent même activement en reformulant des savoirs construits lors de l'étude de L'Étranger :

\section{Extrait 4}

149 Ens : Meursaut est condamné à mort pourquoi est-ce que ça nous parait normal que ce soit Meursaut qui raconte au présent et pas quelqu'un d'autre 150 Af : C'est sa vision à lui

$152 \mathrm{Am}$ : C'est la focalisation interne

153 Autre Am : Il est pas encore mort

[...]

163 Ens : Comme disait Am. c'est le récit en direct

Mais la satisfaction de l'enseignant sera de courte durée. La suite de l'interaction montre que la contrainte induite par ce récit en focalisation interne n'est pas intégrée :

\section{Extrait 5}

178 Ens : Donc notez ça [...] le récit devait être au présent comme dans L'Étranger +

[...]

$180 \mathrm{Au}$ : Monsieur + mardi dernier vous nous avez dit que les deux systèmes étaient possibles

181 Ens : Non + mardi j'ai dit qu'il y avait deux types de récit avec deux régimes temporels $[\ldots]+$ vous devez rayer la première des deux possibilités + reprenez la feuille de route [...] eh s'il VOUS plait ça continue le cours vous êtes d'accord c'était le piège $+K$. on n'écrit pas sur les tables

184 Af: Si on emploie le passé simple c'est faux $\pi$

185 Ens: C'est ce que je suis en train de vous dire + écoutez parce que si personne n'écoute personne ne comprend c'est normal

Les élèves remettent ici en cause la contrainte qui semblait acquise quelques minutes avant. Ils sont moins attentifs et semblent ne pas comprendre 
pourquoi le passé simple n’a pas été explicitement interdit sur la « feuille de route».

\section{Extrait 6}

188 Af : On nous aurait lourdement pénalisés $\boldsymbol{\lambda}$ Comme un hors-sujet $\boldsymbol{\lambda}$ 189 Ens : Non pas comme un hors-sujet mais comme un problème de cohérence + si vous avez choisi et tenu le registre temporel au passé sans faire de faute je pense qu'on ne vous aurait pas lourdement pénalisés [...] + si en plus vous aviez vu ça c'est ce qui fait passer une copie de 13 à 15

L'enseignant s'efforce de rassurer ses élèves mais la suite de l'interaction montre qu'il n'a pas récupéré leur attention. À la fin du cours, Al. déclare par ailleurs brusquement : "Y a pas d'humour!», remettant en cause une des contraintes de la feuille de route, pourtant respectée par la plupart de ses camarades.

Les commentaires sur la séance produits lors des entretiens éclairent le développement chronologique de ce cours.

\section{Analyse d'un conflit de ressources}

\section{Tensions entre développements de la planification et mise en œuvre de principes socioconstructivistes}

L’enseignant, désigné par le prénom de Jeremy, est un jeune agrégé qui n'a pas eu de formation IUFM. C'est sa première année d'exercice en lycée. Si l'on considère, à la suite d'A. Schütz que « l'homme dans son quotidien dispose à tout moment d'un réservoir de connaissances qu'il utilise comme schèmes d'interprétation de ses expériences passées et présentes, et qui détermine aussi ses anticipations sur les choses à venir " (1987: 202), on peut dire que Jeremy, dans l'exercice de son métier, dispose d'un répertoire de schèmes d'interprétation peu étendu. Dans le même temps, on observe une séance planifiée de manière cohérente et sous-tendue par des objectifs et des principes didactiques clairs. L'interaction de classe et l'entretien d'autoconfrontation montrent une attention à la dimension co-construite des savoirs : «Voilà, voilà, c'est le coup de théâtre. Je réussis à leur faire dire... et je fais appel à leur bon sens [...] pour qu'ils disent bon, ben, oui, il y a un problème de récit, ici [...] et ce qui est intéressant, c'est que je ne m'arrête pas là, je fais vraiment ça dans un but de construction du savoir ici » (c6).

Tout semble donc bien se passer, sinon " comme prévu », du moins d'une manière qui doit permettre d'atteindre les objectifs. Mais pour faire admettre la nature indiscutable de la contrainte temporelle, l'enseignant s'éloigne de la dynamique de construction des savoirs dans laquelle il s'était engagé. D'une part, l'attention de la classe se dissipe, on note de nombreux rappels à l'ordre et le recours plus fréquent à des énoncés injonctifs ( $c f$. l'extrait 5). L'enseignant, lors de l'autoconfrontation, commente ce qu'il qualifie 
de «bazar ambiant " : « Je sais pas quand ça s'est dégradé, mais le contraste avec l'attention, l'intérêt du début du cours c'est impressionnant... " (c 9). D'autre part, dès le " coup de théâtre ", le cours devient plus magistral. Les prises de parole de l'enseignant deviennent plus longues, il demande aux élèves de prendre des notes. Le coup de théâtre ressemble à un " coup de force " discursif. L'usage à deux reprises de questions rhétoriques ("Vous êtes d'accord », extraits 2 et 5) censé permettre à l'enseignant de s'assurer de l'adhésion des élèves a en fait une fonction argumentative (" transférer aux conclusions l'accord initialement octroyé aux prémisses ", Amossy 2006 : 14).

On constate alors des remises en cause explicites, de plus en plus fréquentes, des contraintes qui semblaient acquises, depuis la contrainte nouvelle concernant le régime temporel (le récit au présent) jusqu’à celles répertoriées dans la feuille de route (présence d'humour noir). Comment en est-on arrivé là ?

\section{De la légitimité du piège en didactique ?}

Si le piège se définit comme un "artifice pour mettre quelqu'un dans une mauvaise situation» (Le Robert illustré 2000), on peut se demander comment il peut légitimement être utilisé dans un contexte éducatif ou didactique. Pourtant, dans notre corpus, ce terme est employé à trois reprises, par l'enseignant et ses élèves, comme une métaphore pour décrire la situation telle qu'ils l'on vécue.

Cette métaphore du piège fonctionne à deux niveaux. Tout d'abord, le piège du dispositif pédagogique. L'enseignant, qui connait déjà le texte d'Al. a choisi de le "garder comme joker" pour que la réflexion sur la contrainte temporelle parte de l'observation par les élèves de leurs propres productions. Ce piège s'apparente plutôt, du point de vue de la stratégie enseignante, à ce qu'Y. Guégan appelle les « ruses bienveillantes» (2008: 11). Pas forcément bienveillante du point de vue de l'élève qui a pu se sentir instrumentalisé. Jeremy propose d'ailleurs l'analyse suivante : "Al. est un très bon élève et là, visiblement, il est le moteur du bazar ambiant [...] " (c.9). Il reprend cette question lors d'un deuxième entretien réalisé plus tard : " Je voulais les mettre en situation de découverte, plutôt que de leur imposer une consigne, mais il ne fallait pas les mettre en échec et ça je ne le referai pas». L'enseignant formule ici explicitement un principe de son action didactique $^{4}$ : pour conduire les élèves sur le chemin d'une critique collective et constructive de leurs productions il faut leur garantir un cadre interactionnel « sécure ", où ils ne sentent pas leurs faces mises en danger.

4. Principe qui guide déjà implicitement une partie de son action pendant le cours comme le montre ces recommandations aux élèves : « 22 : Et maintenant qui fait la critique négative de F. ? Vas-y Al. mais fais-le gentiment hein s'il te plait + c'est constructif + c'est simplement pour dire à F. comment améliorer son travail. » 
À un deuxième niveau, la métaphore du piège permet de penser ce qui se joue dans les non-dits de la contrainte d'écriture. Le terme de "piège " est utilisé dans l'interaction didactique elle-même (extrait 5, intervention 181, "c'était le piège ») et, lors de l'entretien, dans les commentaires du début du cours ("Et à ce moment-là, la classe, il me semble, est attentive, ils se rendent compte qu'il y avait des pièges à trouver...»). Utiliser le régime temporel du passé, c'était tomber dans un de ces " pièges ".

Pour les élèves, le terme est utilisé également pour penser la situation complexe dans laquelle ils se retrouvent. Ainsi, lors de l'entretien réalisé avec eux, une élève commente ainsi le dysfonctionnement du cours. Elle s'exclame : "on lui avait dit, le mardi, qu'on pouvait pas raconter du point de vue du bourreau, mais il nous a soutenu le contraire! » et Al. surenchérit : "Il nous a piégés, quoi! ». L'emploi que font les élèves du mot piège se rapproche de la définition du dictionnaire Robert et montre que les élèves ne perçoivent pas le choix de Jeremy de ne pas « tout leur dire " comme une stratégie d'enseignement bienveillante.

Les élèves sont en fait placés dans ce qu'on peut considérer comme une "situation problème " : comment conduire un récit dont le narrateur meurt à la fin ? Formuler la difficulté en termes de " franchissement d'obstacles " (voir les travaux sur ces concepts développés en didactique des sciences, à la suite notamment de Astolfi 1992), plutôt qu'en termes de "piège " aurait probablement conduit, en amont (ou en accompagnement) de l'écriture, à identifier explicitement l'obstacle en question et à réfléchir collectivement aux ressources d'écriture dont les élèves pouvaient disposer pour répondre à la consigne 5 . Cela aurait également permis d'éviter à Al. le sentiment d'échec.

\section{Le recours au « on " pour échapper au conflit interpersonnel}

Pour sortir du "piège » qui les mène potentiellement au conflit interpersonnel, l'enseignant, comme les élèves, vont en appeler à une autorité, sinon supérieure, du moins extérieure, qui renvoie à deux instances partiellement distinctes :

1. Celle du concepteur de l'épreuve, potentiel concepteur de tous les sujets à venir, qui apparait dans l'extrait 6 , déjà cité ci-dessus, sous la forme d'un " on » dont l'enseignant se sert comme d'un bouclier énonciatif :

128 Ens : Et la difficulté majeure de ce sujet un peu vicieux [...] c'est qu'on vous demandait de faire parler quelqu'un qui raconte sa propre mort.

140 Ens : Oui mais oublions ça si on vous dit de rédiger à la première personne il faut le faire.

5. Avant de se heurter à la difficulté du régime temporel, Jeremy n'a pas eu de mal, à travers la lecture critique des textes produits par les élèves, à faire découvrir à la classe une autre ressource pour résoudre la situation problème : faire décrire la dernière vignette par le bourreau-narrateur pour faire comprendre son funeste destin. 
En convoquant ce concepteur/énonciateur absent " on ", Jeremy rappelle qu'une des caractéristiques de la forme scolaire est " la répétition d'exercices n'ayant d'autres fonctions que d'apprendre et d'apprendre selon les règles ou, autrement dit, ayant pour fin leur propre fin » (Vincent, Lahire et Thin 1994 : 39). Les limites de la justification des règles semblent ici atteintes. Tout en défendant, dans le cours et dans le commentaire, la cohérence du sujet ainsi que la logique des dits et des non-dits de l'énoncé, Jeremy révèle implicitement qu'il n'en est pas l'auteur et n'en approuve pas forcément toutes les exigences mais qu'il faut néanmoins s'y conformer.

2. Celle de l'évaluateur, qui corrigera l'épreuve à l'examen. Dans l'extrait 6 , les élèves demandent : " on nous aurait lourdement pénalisés ", convoquant eux aussi un personnage absent : l'évaluateur qui les corrigera le jour de l'examen. Ce peut être une manière de sortir du face-à-face avec leur enseignant, de lui rappeler que quelqu'un d'autre jugera leurs productions. Mais c'est aussi sans doute une manière de solliciter l'enseignant pour qu'il les rassure, en leur répondant comme membre et représentant de la communauté des évaluateurs au baccalauréat. Celui-ci, forcément inexpérimenté en la matière, répond cependant en ayant sans doute en tête les Programmes officiels, qui stipulent que le terme d'invention «ne renvoie pas obligatoirement à des capacités d'imagination mais doit montrer la capacité à trouver des arguments et à mettre en ouvre un style efficace au sein d'un discours rhétorique ".

L'ambigüité de cette épreuve a suscité de vives réactions au sein des collègues de l'équipe Diltec-Idap où le corpus a été discuté. L'écriture créative ne trouve-t-elle pas son intérêt dans la capacité à se jouer de règles explicites ou implicites? Et dans le même temps, peut-on évaluer des productions d'élèves sans se référer à des règles qu'ils doivent avoir intégrées ? Et qui peut décider celles qui sont «transgressables » et celles qui ne le sont pas $^{6}$ ? Ces questions nous rappellent la nécessité de situer l'action d'enseigner dans un contexte plus large, ici le contexte institutionnel des épreuves du baccalauréat, et au-delà le contexte du champ de l'écriture littéraire. Elles posent des problèmes didactiques non résolus, véritables défis que ce jeune enseignant doit relever dans ce cours.

Ces analyses nous ont permis de suivre un enseignant contraint à mobiliser d'autres ressources que celles prévues dans la planification de son cours. Il se retrouve rapidement pris dans des dilemmes du métier d'enseignant que sa relative inexpérience ne lui donne pas les moyens de résoudre facilement ni rapidement. Les ressources (planification du coup de théâtre et principe socioconstructiviste) qu'il met en œuvre pour tenter d'atteindre coute que coute les objectifs qu'il s'était fixés, entrent en conflit les unes avec les autres. Le double piège tendu par la consigne d'écriture et par le dispositif

6. Ainsi, Guy de Maupassant (La nuit) à Anouar Benmalek (O Maria, Fayard, 2006), les exemples d'auteurs qui s'autorisent à faire parler un narrateur mort ne manquent pas dans la littérature, dans des genres littéraires bien sûr différents de celui attendu des élèves. 
pédagogique pourrait se refermer sur le cours mais les participants en desserrent la mâchoire en convoquant des autorités-énonciatrices absentes et, derrières elles, toute l'institution scolaire. Celle-ci, en effet, porte la responsabilité des risques d'ambigüité que comporte l'épreuve d'écriture d'invention. Et le recours à cette instance permet un ménagement réciproque des faces et le dépassement du conflit. Même si la fin de la séance est moins consensuelle que son début, les séances suivantes verront se poursuivre les apprentissages qui n'ont pu se construire exactement comme prévu lors de ce cours.

\section{Un corpus qui nous éclaire sur les processus de construction du répertoire didactique}

Un tel corpus présente des atouts pour être utilisé en formation initiale. Certes on peut discuter certains choix de l'enseignant (notamment le fait d'imposer un seul régime temporel ou, si on accepte cette contrainte, le fait de ne la faire formuler qu'après le premier jet d'écriture). Mais, globalement, la qualité de la séance planifiée permet de concentrer l'analyse sur le développement des échanges dans la classe, sur les pratiques interactionnelles au cœur du métier et non sur la fiche de préparation (question qui vient souvent parasiter l'analyse des pratiques enseignantes vidéoscopées). Le fait que le cours rencontre des difficultés aiguise le regard. De même que les malentendus dans une interaction, permettent de s'interroger sur le fonctionnement des processus de compréhension, les perturbations que connait le cours rendent saillants certains fonctionnements, de l'interaction et permettent de creuser, avec des stagiaires, la question des conditions pour qu'un échange constructif se développe avec les élèves.

Pour des enseignants en formation initiale le corpus n'offre donc pas un exemple de «bonnes pratiques à imiter», celles-ci pouvant, en formation, se révéler inhibitrices et donc contreproductives. C'est la capacité réflexive de l'enseignant qui est le modèle à suivre ${ }^{7}$. À côté des points positifs qu'il a pu relever (grande attention des élèves dans la première moitié du cours, dynamique de co-construction, acquis des cours précédents que les élèves mobilisent), il constate lui-même certains points faibles : glissement noncontrôlé de la séance vers un cours magistral, dispositif qui a mis en échec le jeune Al., piège de la digression - initiée par les élèves - sur l'évaluation à l'examen. Il interroge ses choix, tire des leçons pour l'avenir, enrichit consciemment ses schèmes d'interprétation et son répertoire d'action et construit là, sous nos yeux, un répertoire didactique dynamique et ouvert (Cicurel $2011:$ 149).

\section{Christiane DESCIMON \& Violaine BIGOT}

7. Le corpus complet peut être consulté sur [http://www.interactions-didactiques.org/]. 


\section{Références bibliographiques}

- AMOSSY, R. (2006). L'Argumentation dans le discours. Paris : Armand Colin.

- ASTOLFI, J.-P. (1992). Apprendre par franchissement d'obstacles ? Repères, 5, 103-116.

- CICUREL, F. (2011). Les Interactions dans l'enseignement des langues. Agir professoral et pratiques de classe. Paris : Didier.

- FILLIETAZ, L. (2005). Mise en discours de l'agir et formation des enseignants : quelques réflexions issues des théories de l'action. In F. Cicurel \& V. Bigot (dir.), Le français dans le monde. Recherches et applications, $\mathrm{n}^{\circ}$ spécial : "Les Interactions en classe de langues : contextes, ressources, enjeux" (pp. 20-31). Paris : CLE international \& FIPF.

- GUÉGAN, Y (2008). Les Ruses éducatives. Paris : ESF.

- SCHÜTZ, A. (1987). Le Chercheur et le quotidien. Phénoménologie des sciences sociales. Paris : Klincksieck.

- VINCENT, G., LAHIRE, B. \& THIN, D. (1994). Sur l'histoire et la théorie de la forme scolaire. In G. Vincent (dir.), L'Éducation prisonnière de la forme scolaire (pp. 11-47). Lyon : Presses universitaires de Lyon.

- VION, R. (1992). La Communication verbale. Paris : Hachette. 